\title{
mTOR Signaling: The Interface Linking Cellular Metabolism and Hepatitis B Virus Replication
}

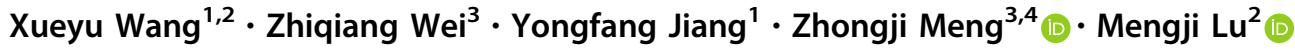

Received: 5 May 2021 / Accepted: 24 August 2021 / Published online: 28 September 2021

(C) The Author(s) 2021

\begin{abstract}
Mammalian target of rapamycin (mTOR) is a conserved Ser/Thr kinase that includes mTOR complex (mTORC) 1 and mTORC2. The mTOR pathway is activated in viral hepatitis, including hepatitis B virus (HBV) infection-induced hepatitis. Currently, chronic HBV infection remains one of the most serious public health issues worldwide. The unavailability of effective therapeutic strategies for HBV suggests that clarification of the pathogenesis of HBV infection is urgently required. Increasing evidence has shown that HBV infection can activate the mTOR pathway, indicating that HBV utilizes or hijacks the mTOR pathway to benefit its own replication. Therefore, the mTOR signaling pathway might be a crucial target for controlling HBV infection. Here, we summarize and discuss the latest findings from model biology research regarding the interaction between the mTOR signaling pathway and HBV replication.
\end{abstract}

Keywords Mammalian target of rapamycin (mTOR) · Hepatitis B virus (HBV) · Metabolism · Autophagy

\section{Introduction}

Hepatitis B virus (HBV) infection remains a global threat to public health. Patients with chronic HBV infection are prone to cirrhosis, liver failure, and hepatocellular carcinoma (HCC) (Tang et al. 2018). In the past few decades, effective vaccines have been widely used to reduce the incidence of $\mathrm{HBV}$ infection, especially in newborns. However, the current therapeutic strategies for HBV infection using pegylated interferon and nucleos(t)ide

Mengji Lu

mengji.lu@uni-due.de

Zhongji Meng

zhongji.meng@taihehospital.com

1 Department of Infectious Diseases, The Second Xiangya Hospital, Central South University, Changsha 410011, China

2 Institute of Virology, University Hospital Essen, University of Duisburg-Essen, 45122 Essen, Germany

3 Institute of Biomedical Research, Hubei Clinical Research Center for Precise Diagnosis and Treatment of Liver Cancer, Taihe Hospital, Hubei University of Medicine, Shiyan 442000, China

4 Department of Infectious Diseases, Taihe Hospital, Hubei University of Medicine, Shiyan 442000, China analogues have limited efficiency against the disease (Liang et al. 2015; Subic and Zoulim 2018; Fanning et al. 2019).

HBV, which belongs to the Hepadnaviridae family, is an enveloped virus with a double-stranded DNA genome of a size of $3.2 \mathrm{~kb}$, and is thus one of the smallest DNA viruses currently known (Karayiannis 2017). HBV replicates through reverse transcription (Glebe and Bremer 2013; Tong and Revill 2016). HBV covalently closed circular (ccc) DNA copies are formed in the nucleus, then serve as templates for viral transcription, and generate five major RNA molecules. These molecules include $3.5-\mathrm{kb}$ pregenomic (pg) RNA and preC RNA, 2.4- and 2.1-kb preS/S mRNAs, and 0.7-kb HBx mRNA, which encode core/polymerase proteins, precore protein, large envelope protein, middle and small envelope proteins, and $\mathrm{X}$ protein, respectively (Gish et al. 2015; Nassal 2015). Subsequently, the core protein, pgRNA, and viral DNA polymerase are recruited to form nucleocapsids (NCs). In the process of virion release, NCs can directly be assembled with glycoprotein envelope and trigger the secretion of nascent virions, or be reintroduced into the nucleus for amplification of the cccDNA pool (Guo et al. 2007a; Kock et al. 2010). Noninfectious subviral particles (SVPs) composed of three envelope proteins, S-, M-, and L- HBV surface antigen 
(HBsAg), are independently secreted from host cells only through the endoplasmic reticulum (ER)-Golgi secretion pathway.

The life cycles of many viruses can be regulated by the mammalian target of rapamycin (mTOR) signaling pathway (Ranadheera et al. 2018; Bossler et al. 2019). The mTOR signaling pathway is a key regulator of many cellular processes including cell metabolism, growth, proliferation, survival and immunity (Laplante and Sabatini 2012; Liu Y et al. 2015; Saxton and Sabatini 2017), and is abnormally overactivated in numerous human cancers (Ciuffreda et al. 2010; Forbes et al. 2011). Moreover, the mTOR signaling pathway is activated during infection by many viruses, including HBV. Previous studies have shown that the interaction of mTOR signaling pathway and HBV life cycle is complex. While HBV proteins like the $\mathrm{HBV} X(\mathrm{HBx})$ protein are able to modulate this pathway (Zhu et al. 2017; Wang X et al. 2019). HBV replication and gene expression are also regulated by the mTOR signaling pathway (Guo et al. 2007b; Zhang et al. 2011; Huang et al. 2014; Li et al. 2015; Lin et al. 2017; Wang et al. 2020a, 2020b). In addition, the mTOR signaling pathway restricts or degrades viral particles and SVPs in a lysosome-dependent manner (Lin et al. 2019c; Wang et al. 2020b). In this review, we summarize and discuss the major findings in this field.

\section{mTOR Signaling Pathway}

The mTOR protein is an evolutionarily conserved Ser/Thr kinase in the phosphoinositide 3-kinases (PI3K)-related kinase (PIKK) family. mTOR is the catalytic subunit of two complexes, mTORC1 and mTORC2 (Wullschleger et al. 2006); the localization of these complexes in different subcellular compartments affects their activation and function (Betz and Hall 2013).

mTORC1 is a rapamycin-sensitive companion of mTOR that acts as a sensor for growth factors, pressure, energy status, oxygen, and amino acids to control many major cellular processes, including glucose homeostasis, lipid synthesis, and autophagy (Fang et al. 2001; Kim et al. 2002; Hay and Sonenberg 2004). The heterodimer composed of tuberous sclerosis 1 (TSC1) and 2 (TSC2) is a key upstream regulator of $\mathrm{mTORC} 1$. The GTP-bound form of Ras homolog enriched in brain (Rheb) directly interacts with mTORC1 and strongly stimulates its kinase activity. However, TSC1/2 negatively regulates mTORC1 activity by reversing Rheb into its inactive GDP-bound state (Inoki et al. 2003a; Tee et al. 2003). Many upstream signals including growth factors that regulate mTORC1 through the PI3K and Ras pathways, are transmitted through the TSC1/2 complex. Effector kinases, including protein kinase B (PKB/Akt) (Chen et al. 2018), extracellular signal-regulated kinase 1/2 (ERK1/2) (Ma et al. 2005), and p90 ribosomal S6 kinase (RSK) (Roux et al. 2004), can directly phosphorylate and inactivate the TSC $1 / 2$ complex, thereby activating mTORC1. Amino acids also activate mTORC1. Under conditions with sufficient amino acid supply, mTORC1 is activated via localization with the Ragulator-Rag complex (RagA/B and RagC/D) on the lysosome surface after activating Rheb (Efeyan et al. 2012; Groenewoud and Zwartkruis 2013). The activation of mTORC1 can enhance protein or lipid synthesis by modulating the translation regulator $\mathrm{p} 70$ ribosomal S6 kinase 1 (S6K1) and the eukaryotic translation initiation factor $4 \mathrm{E}$ binding protein 1 (4EBP1) or sterol regulatory elementbinding protein 1/2 (SREBP1/2) (Duvel et al. 2010; Wang et al. 2011; Guo et al. 2019), respectively. Han et al. proved that mTORC1 also regulates the trafficking and maturation of SREBP1 through CREB-regulated transcription coactivator 2 (CRTC2). Under nutrient-rich conditions or insulin stimulation, mTOR activation leads to the phosphorylation and activation of SREBP1, thereby enhancing lipogenesis (Han et al. 2015).

The signaling pathway triggered by mTOR competes with other regulators of cellular metabolisms, such as AMP-activated protein kinase (AMPK), another major metabolic sensor. AMPK is activated in response to the increased AMP/ATP ratio, for example, after starvation of glucose. Importantly, AMPK directly or indirectly inhibits mTOR activity (Inoki et al. 2003b; Gwinn et al. 2008). Mitochondria biogenesis and turnover mediated by mTOR and AMPK are essential for regulating the cellular metabolism, especially mitochondria. Peroxisome proliferatoractivated receptor (PPAR) gamma coactivator $1 \alpha(\mathrm{PGC} 1 \alpha)$ is a nuclear cofactor that plays a key role in mitochondrial biogenesis, oxidative metabolism, and gluconeogenesis. A report showed that nuclear mTORC1 controls the transcriptional activity of $\mathrm{PGC} 1 \alpha$ by changing its physical interaction with another transcription factor Yin Yang 1 (YY1) in skeletal muscle (Cunningham et al. 2007). However, AMPK directly phosphorylates and activates PGC1 $\alpha$ in skeletal muscle (Jager et al. 2007). Additionally, under glucose starvation conditions, inactivation of mTORC1 is accompanied with increased expression of PGC1 $\alpha$ in hepatoma cells (Wang et al. 2020a). Consistent with this finding, mTORC1 reduces the fasting-induced activation of PPAR $\alpha$ (Sengupta et al. 2010).

In addition, mTORC1 enhances glycolysis and glucose uptake by regulating the transcription factor hypoxia-inducible factor (HIF1) (Semenza 2010) and promotes cell growth by negatively regulating autophagy, which is necessary for the recycling of damaged organelles and the adaptation of organisms to nutrient starvation (Kim et al. 2011). In mammals, mTORC1 directly phosphorylates and 
inhibits the ULK1/Atg13/FIP200 complex, which is an essential kinase complex that initiates autophagy. Activating mTORC1 can inhibit lysosome function by inhibiting the activity of transcription factor EB (TFEB), the master regulator of lysosomal biogenesis. Nutritional starvation or inhibition of mTORC1 activates TFEB by promoting its nuclear translocation, thereby promoting autophagic flux (Zhou et al. 2013).

On the other hand, mTORC2 is a rapamycin-insensitive companion of mTOR (Jacinto et al. 2004; Sarbassov et al. 2004) and can be directly activated by PI3K (Shimobayashi and Hall 2014). Interestingly, long-term rapamycin treatment can also inhibit mTORC2 in some cell types, which may be caused by inhibition of mTORC 1 and reduction in binding of mTORC1 to the nascent mTORC 2 complex (Delgoffe et al. 2011). Compared with the mTORC1 pathway, the mTORC2 pathway is much less understood. The downstream genes of mTORC2 include several members of the AGC kinase subfamily, such as $A k t$, serum and glucocorticoid-induced protein kinase 1 (SGKl) and protein kinase $\mathrm{C}-\alpha(P K C-\alpha)$. mTORC2 directly activates Akt by phosphorylating the hydrophobic motif (Ser473) of Akt, which is required for the maximum activation of Akt (Sarbassov et al. 2005). Akt regulates cellular metabolism, survival, apoptosis, growth and proliferation through phosphorylation of several effectors.

\section{HBV-Mediated Activation of mTOR Signaling Pathway}

mTOR signaling is a key regulator of many cellular processes including metabolism, proliferation and survival. As a consequence, the life cycles of numerous viruses are regulated by the mTOR signaling pathway (Ranadheera et al. 2018; Bossler et al. 2019). Notably, chronic HBV infection is one of the key factors in the development of HCC. Guo et al. demonstrated that HBV RNA transcription and subsequent DNA replication are inhibited by Akt, an upstream factor of mTORC1, in HBV-transfected cells (Guo et al. 2007b). These inhibitory effects appear to be mediated by mTORC1 activation because this inhibition can be reversed by rapamycin. In addition, PI3K, Akt and mTOR inhibition using high concentrations of drugs can promote HBV RNA transcription and DNA replication in vitro. Consistent with these findings, HBsAg expression in chronic hepatitis B is significantly upregulated compared with that in HBV-associated HCC (Wang M et al. 2013), which has a higher PI3K/Akt activity. However, low inhibitor concentrations of PI3K, Akt and mTOR mainly modulate posttranscriptional steps of HBV life cycle (Lin et al. 2020). The roles of HBV in the mTOR signaling pathway are summarized in Fig. 1.

\section{Role of HBsAg in the mTOR Signaling Pathway}

The HBV entry process may activate the Akt/mTOR signaling pathway (Xiang and Wang 2018). However, shortterm treatment with Akt inhibitors does not block HBV entry, suggesting that Akt activation induced by HBV infection is not essential for the viral entry process. Yet, it is not clear how the activation of the Akt/mTOR pathway is triggered. It is reported that uptake of various ligands or virus particles may activate the cellular Akt/mTOR pathways (Ji and Liu 2008; Eaton et al. 2014). This process needs to be examined in the future. HBV virions and noninfectious SVPs contain three glycosylated HBV surface antigens, S-, M-, and L-HBsAg (Mehta et al. 1997; Sigrid Schmitt 1999). HBV infection can induce the synthesis of a large quantity of viral proteins and lead to the accumulation of HBsAg in the ER lumen to trigger ER stress, thereby activating the PI3K/Akt/mTOR pathway (Choi et al. 2019). L-HBsAg has been shown to activate the PI3K/Akt/mTOR pathway to promote tumorigenesis (Liu et al. 2011). Consistent with this evidence, Teng et al. reported that wild type or mutant pre-S proteins can activate mTOR in Huh7 cells (Teng et al. 2011). It has also been reported that pre-S1 deletions lead to accumulation of $\mathrm{L}-\mathrm{HBsAg}$, thereby triggering ER stress to activate the mTOR signaling pathway (Choi et al. 2019). The pre-S mutant proteins can induce ER stress, leading to formation of ground glass hepatocytes (GGHs) (Wang et al. 2003). In addition, L-HBsAg and pre-S2 mutants activate the mTOR signaling pathway through induction of ER stress-dependent vascular endothelial growth factor (VEGF) A and Akt activation, resulting in aberrant glucose uptake and lactate production in tumorigenic processes. In accordance with this finding, the pre-S2 mutant proteins also trigger ER stress-mediated VEGF/Akt/mTOR and NF- $\mathrm{KB} / \mathrm{COX}-2$ signaling pathways to induce cell inflammation and transformation (Hung et al. 2004; Yang et al. 2009). Moreover, pre-S mutant-induced ER stress may also modulate the activity of SREBP1, ATP citrate lyase (ACLY), and fatty acid desaturase 2 (FADS2) (Teng et al. 2015b), major regulators of lipid metabolism, by activating the Akt/ mTOR signaling pathway (Yang et al. 2009). Interestingly, activation of mTOR can inhibit HBsAg synthesis by promoting the interaction between histone deacetylase 1 (HDAC1) and the transcription factor YY1, which binds to the pre-S1 promoter (Teng et al. 2011). Disruption of HDAC1 eliminates the inhibitory effect of mTOR on pre-S transcription.

Collectively, the evidence indicates that wild type and mutant HBsAg accumulation can trigger ER stress and activate the Akt/mTOR signaling pathway. This process may promote tumorigenesis and is considered as an 


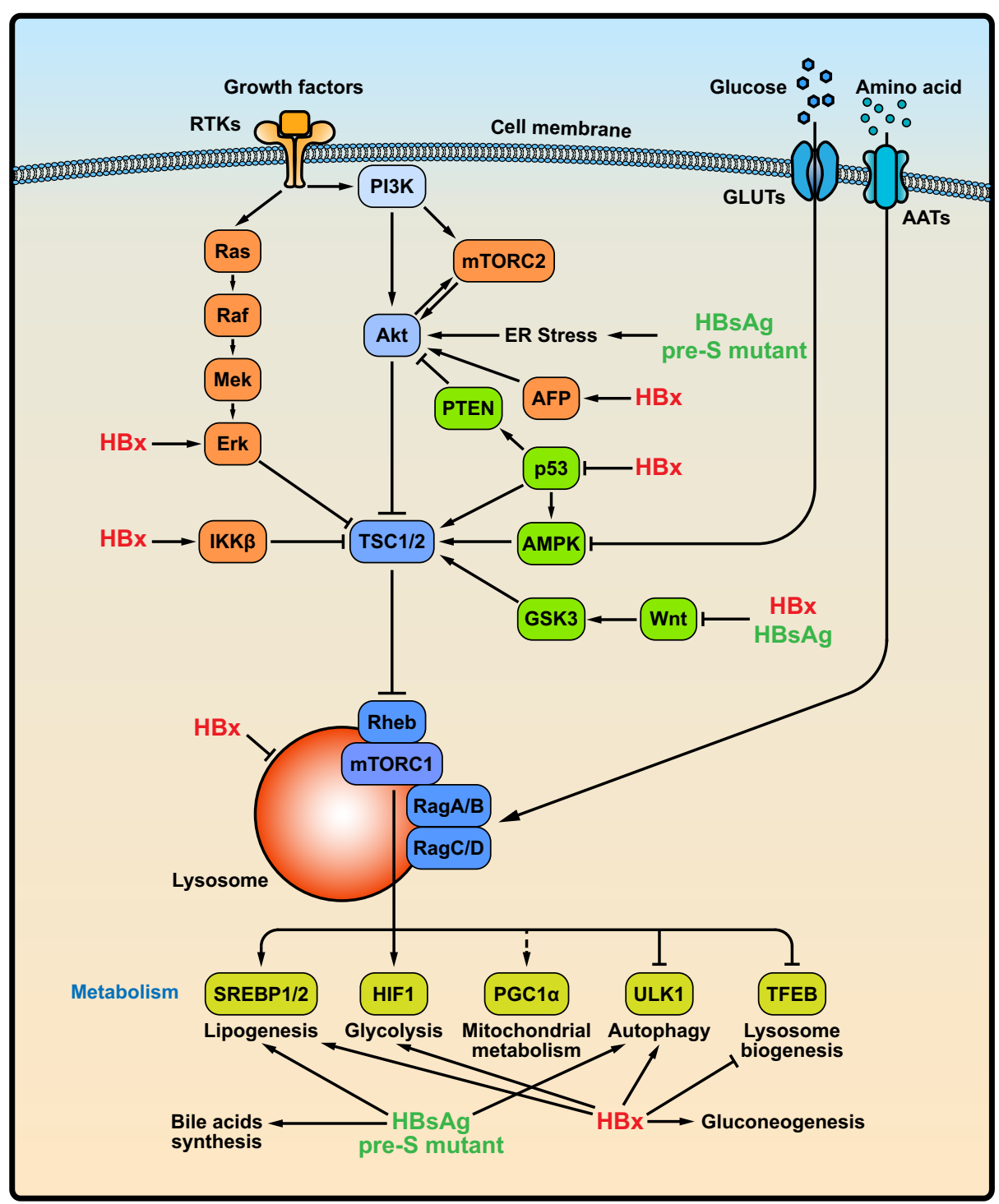

Fig. $1 \mathrm{HBV}$ infection activates the PI3K/Akt/mTOR signaling pathway. Growth factors and nutrition, including glucose and amino acids, are factors known to activate the PI3K/Akt/mTOR signaling pathway. During the HBV infection process, HBsAg and pre-S mutant protein may accumulate in the ER lumen to trigger ER stress, thereby activating the PI3K/Akt/mTOR signaling pathway. In addition, HBsAg and HBx indirectly activates the mTOR signaling by regulating Wnt/ $\beta$-GSK3 pathway. HBx-mediated activation of the RAS/RAF/MAPK pathway and IKK $\beta$ induces the mTOR signaling pathway by inhibiting the activity of TSC1/2. Additionally, $\mathrm{HBx}$

underlying mechanism of HBV-associated hepatocarcinogenesis. However, whether and how HBV may benefit from the HBsAg-induced ER stress remains elusive. Our recent approach using low doses of tunicamycin, a glycosylation inhibitor, showed that even artificially triggered ER-stress strongly promotes HBV replication and production by modulating viral assembly and release (unpublished data). indirectly modulates $\mathrm{p} 53$, AFP, or Wnt/ $\beta$-GSK3 pathway to activate the mTOR signaling pathway. More importantly, HBV infection interferes with hepatic metabolism signaling pathways, including glucose homeostasis and lipid metabolism pathways. Abbreviations: GLUTs, glucose transporters; AATs, amino acid transports; RTKs: receptor tyrosine kinases; IKK $\beta$, IкB kinase $\beta$; AFP, alpha fetoprotein; PTEN, phosphatase and tensin homolog; SREBP1/2, sterol regulatory element-binding protein $1 / 2 ; \operatorname{PGC} 1 \alpha, \operatorname{PPAR} \gamma$ coactivator $1 \alpha$; HIF1, hypoxia-inducible factor; TFEB, transcription factor EB.

\section{Role of HBx in the mTOR Signaling Pathway}

The HBx protein, the product of the smallest of the four overlapping open reading frames of the HBV genome, plays a vital role in $\mathrm{HBV}$ replication and the pathogenesis of HBV-associated HCC. HBx transfection in hepatoma cells increases the expression of mTOR (Wang $\mathrm{P}$ et al. 2013), and enhance the formation of autophagosomes and autolysosomes through the $\mathrm{PI} 3 \mathrm{~K} / \mathrm{Akt} / \mathrm{mTOR}$ signaling 
pathway (Wang P et al. 2013; Wang et al. 2014). HBX may also mediate the RAS/RAF/MAPK pathway activation, thereby inducing the mTOR signaling pathway by inhibiting the activity of TSC1/2 (Tarn et al. 2001; Chung et al. 2004). In addition, mTOR activation by HBx appears to be dependent on I $\mathrm{KB}$ kinase $\beta$ (IKK $\beta$ ), a major downstream kinase in the TNF $\alpha$ signaling pathway. IKK $\beta$ physically interacts with and phosphorylates TSC1 at Ser487 and Ser511, which results in TSC1 suppression and consequent mTOR activation (Lee et al. 2007). In addition, in an HBx transgenic (Tg) mouse model and HBV-associated HCC hepatocytes, HBx expression can regulate the IKK $\beta / \mathrm{mTOR} / \mathrm{S} 6 \mathrm{~K} 1$ signaling pathway (Yen et al. 2012). Disruption of IKK $\beta$ reverses HBx-mediated S6K1 activation, cell proliferation and VEGF production. Furthermore, HBx upregulates the expression of AFPR and AFP, which are early indicators of $\mathrm{HBx}$-driven hepatocarcinogenesis, to activate the PI3K/Akt/mTOR signaling pathway (Zhu et al. 2015, 2017). Moreover, the HBx-induced antiapoptotic mechanism is essential for promoting the malignant transformation of hepatocytes via activation of the PI3K/ Akt/mTOR signaling pathway (Wang $X$ et al. 2019). The weakness of these studies is that the expression levels of $\mathrm{HBx}$ protein in transfected cells may be higher than that during $\mathrm{HBV}$ infection and replication and cause nonphysiological effects.

The existing evidence suggests that $\mathrm{HBx}$ binds to and inactivates the transcription factor and tumor suppressor p53. Chung et al. reported that HBx disrupts p53-mediated transcription of PTEN, which is a negative regulator of the PI3K/Akt/mTOR signaling pathway (Chung et al. 2003). Consistent with this report, $\mathrm{HBx}$ can inhibit the expression (Lee and Rho 2000) or change the promoter binding site of p53 (Chan et al. 2013, 2016), which has been proven to inhibit the mTOR signaling pathway via activation of AMPK or TSC1/2 (Feng et al. 2005). Moreover, HBV proteins, including $\mathrm{HBsAg}$ and $\mathrm{HBx}$, activate $\mathrm{Wnt} / \beta$-catenin pathway (Cha et al. 2004; Lee et al. 2005; Daud et al. 2017) to inhibit GSK3 and TSC2, thereby promoting the mTOR signaling pathway (Inoki et al. 2006).

Altogether, the evidence indicates that HBx may indirectly activate the PI3K/Akt/mTOR signaling pathway, which plays a vital role in the development and progression of HBV-associated HCC. Though the reports mentioned above indicate an important role of $\mathrm{HBx}$ in the activation of mTOR signaling pathway, the findings are rather diverse and could not be integrated into a clearly unified concept. Future studies are required to find the connections between the events described in these studies.

\section{Role of HBV Infection in mTOR-Mediated Metabolism}

The liver is an important organ for metabolic processes and plays key roles in glucose homeostasis and lipid metabolism. During HBV infection, many cellular signal transduction pathways are altered, including the PI3K/Akt/ mTOR pathway (Teng et al. 2011, 2015a; Xu et al. 2013; Rawat and Bouchard 2015). The mTOR signaling pathway not only plays an essential role in coordinating anabolism and catabolism at the cellular level, but also has an important function in metabolic regulation in organisms (Saxton and Sabatini 2017).

With the discovery of a major bile salt carrier human sodium taurocholate cotransport peptide (hNTCP) as a functional receptor for $\mathrm{HBV}$, the role of $\mathrm{HBV}$ in cellular metabolism has been placed at the forefront. Yan et al. showed that HBV pre-S1 may interfere with the physiological function of hNTCP to block bile acid uptake (Yan et al. 2014; Cheng et al. 2019). Decreased uptake of bile acid could promote compensatory bile acids synthesis and increase cholesterol supply to maintain homeostasis (Geier 2014; Patman 2014). In a transplant mouse model with HBV infection, an increased level of human cholesterol $7 \alpha-$ hydroxylase (CYP7A1), the rate-limiting enzyme that converts cholesterol to bile acids, was shown, consistent with decreased nuclear translocation of farnesoid $\mathrm{X}$ receptor (FXR, the positive transcription factor of SHP), and significantly reduced levels of SHP, the corepressor of hCYP7A1 transcription (Oehler et al. 2014).

HBV infection has been shown to regulate gluconeogenesis and aerobic oxidation of glucose. Park et al. showed that $\mathrm{HBx}$ acts as a positive regulator of gluconeogenesis (Shin et al. 2011). Increased HBx expression upregulates the expression of the key gluconeogenesis enzymes PEPCK and G6Pase and the production of hepatic glucose, leading to hyperglycemia and impaired glucose tolerance in $\mathrm{HBx} \mathrm{Tg}$ mice. In addition, $\mathrm{HBV}$ pre-S2 mutants can induce aerobic oxidation of glucose by activating the mTOR signaling pathway (Teng et al. 2015a). HBV infection stimulates the expression of G6PD, the first and rate-limiting enzyme of the pentose phosphate pathway (PPP), through HBx-mediated nuclear factor erythroid 2-related factor 2 (Nrf2) activation (Liu B et al. 2015).

In addition, mTORC1 activation stimulates de novo lipogenesis by interacting with PPAR $\gamma(\mathrm{Li}$ et al. 2014) and regulating the activity of SREBP1, two master regulators of lipid metabolism in hepatocytes (Peterson et al. 2011). It has been reported that $\mathrm{HBx}$ expression leads to lipid accumulation in hepatocytes mediated by SREBP1 and PPAR $\gamma$ (Kim et al. 2007). A number of studies have consistently demonstrated that HBV infection has an impact on 
lipid metabolism. Fatty acid binding protein 5 (Fabp5), acyl-CoA binding protein (ACBP), SREBP2, ACLY, and fatty acid synthase (FAS), which are involved in fatty acid metabolism and synthesis, are strongly upregulated in $\mathrm{HBX}$ $\mathrm{Tg}$ mice (Yang et al. 2008). HBV is an enveloped virus, and its successful assembly and secretion depend on the biogenesis of lipids and the formation of lipid membranes. HBx mediates the transcription of SREBP1, FAS, and PPAR $\gamma$ by activating lipogenesis induced by the nuclear receptor LXR $\alpha$ in HBV-associated HCC (Kim et al. 2008; $\mathrm{Na}$ et al. 2009). Furthermore, pre-S2 mutant-mediated induction of mTOR can activate SREBP1/ACLY/FAS, thereby inducing lipid accumulation (Teng et al. 2015b). On the other hand, HBV infection is associated with reduced prevalence of fatty liver, hypertriglyceridemia and metabolic syndrome in patients (Wong et al. 2012). This observation may have different interpretations and needs to be analyzed further.

Taken together, HBV infection interferes with hepatic metabolism pathways, including glucose homeostasis and bile acid and lipid metabolism pathways, and eventually leads to metabolic disorders. Alteration of these metabolic pathways may also contribute to the pathological process of HBV-associated HCC. On the other hand, viral infections require active metabolisms to supply energy and building blocks (Claus and Liebert 2014). HBV life cycle is also strongly dependent on host cell metabolism and its regulation (also see Conclusion). There are still many aspects in this direction for future research, for example, how different metabolic pathways and metabolites influence HBV replication via mTOR signaling pathways. Therefore, studies on the metabolic pathways during HBV infection could provide new insights and therapeutic approaches.

\section{mTOR Inhibits HBV Replication}

The activation of mTOR by HBV infection can enhance cellular metabolism, autophagy, and immune responses to reduce the damage to host cells. Although the PI3K/Akt/ mTOR pathway benefits most viruses, the PI3K/Akt/ mTOR pathway has been shown to reduce HBV replication in some experimental conditions and may be partly responsible for low or absent HBV replication in transformed and tumor cells. Specifically, triggering the PI3K/ Akt/mTOR signaling pathway directly inhibits the transcription of HBV 3.5-kb and 2.4-kb RNA and HBV replication in hepatoma cells (Guo et al. 2007b). In addition, mTOR can inhibit HBV replication by preventing the recruitment of the YY1-HDAC1 complex to the pre-S1 promoter (Teng et al. 2011). However, the interaction between HBV replication and mTOR-regulated cellular processes is far more complex.

The mTOR kinase complex negatively regulates autophagy initiation and its inhibition activates the ULK kinase complex association with the PIK3C3/BECN1/ ATG14 complex, leading to the formation of autophagosomes. Then, autophagosomes fuse with lysosomes to degrade cytoplasmic cargo (Fig. 2, bottom), whose activity also negatively regulated by the mTOR kinase (Lin et al. 2019c; Wang et al. 2021). Studies have found that autophagosome formation is essential for efficient HBV replication in different cell and animal model (Sir et al. 2010; Li et al. 2011; Tian et al. 2011; Lin et al. 2019a; Lin et al. 2019b; Lin et al. 2019c; Wang J et al. 2019; Wang et al. 2020a; Wang et al. 2020b). In contrast, autophagy inhibition can prevent HBV replication both in vivo and in vitro.

Recent studies demonstrated that HBV replication and assembly can be promoted by initiating the early phase of autophagy. However, a great part of HBV antigens and virions is degraded during the late phase of autophagy. Sir et al. showed that HBV DNA replication is markedly reduced by the PtdIns3K inhibitor 3-methyladenine (3-MA) or siRNA that targets the PtdIns $3 K$ or ATG7 gene to disrupt the initiation of autophagy (Sir et al. 2010). The noncoding microRNA-99 family targets Akt and mTOR to induce autophagy, thereby promoting HBV replication (Lin et al. 2017). Consistent with these findings, our previous study found that treatment with glucosamine (Lin et al. 2019c) or disruption of $O$-GlcNAcylation by OSMI-1 or siRNA that targets OGT greatly enhances HBV replication by initiating the early phase of autophagy by inhibiting mTOR signaling pathway (Wang et al. 2020b). Moreover, autophagy is initiated by inhibition of the Akt/mTOR signaling pathway at low glucose concentrations, with enhanced HBV replication at the same time (Wang et al. 2020a). Our recent study demonstrated that HBV inhibited lysosomal activity is restored to enhance HBV antigens degradation by inhibiting Akt/mTOR signaling after silencing CCDC88A in hepatoma cells with HBV replication (Wang et al. 2021). Some studies have reported that certain drugs, such as rapamycin (an mTOR inhibitor), cisplatin and dexamethasone, induce the initiation of autophagy and promote HBV replication by inhibiting the mTOR signaling pathway (Huang et al. 2014; He et al. 2018; Chen et al. 2019).

The critical role of autophagy in HBV replication has not only been demonstrated in HBV cell culture models but also in vivo. Tian et al. showed that the serum HBV DNA levels are significantly reduced and the HBV DNA replication intermediates were almost undetectable in $\mathrm{HBV}$ transgenic mice if the Atg 5 gene is specifically knocked out in the liver (Tian et al. 2011). Our recent study illustrated 
Fig. 2 mTOR-mediated host responses regulate HBV RNA transcription, assembly and secretion of virions and subviral particles. Top: mTOR and AMPK, two major metabolic sensors, control the activity of PPAR $\alpha$ and PGC1 $\alpha$ to regulate HBV transcription through modulating HBV enhancers and promoters. Bottom: mTOR inhibits both the early (autophagosome formation) and late (lysosomal activity) phases of autophagy. HBsAg utilizes early autophagic structures for envelope formation. HBV virions and subviral particles are degraded during the late phase of autophagy. Abbreviations: PPAR $\alpha$, peroxisome proliferator-activated receptor alfa; PGC1 $\alpha$, PPAR $\gamma$

coactivator $1 \alpha$; FXR $\alpha$, farnesoid $\mathrm{X}$ receptor $\alpha$; RXR, retinoid $\mathrm{X}$ receptor alpha; LRH1, liver receptor homolog $1 ; \mathrm{HNF} 4 \alpha$, hepatocyte nuclear factor $4 \alpha$; TFEB, transcription factor EB.

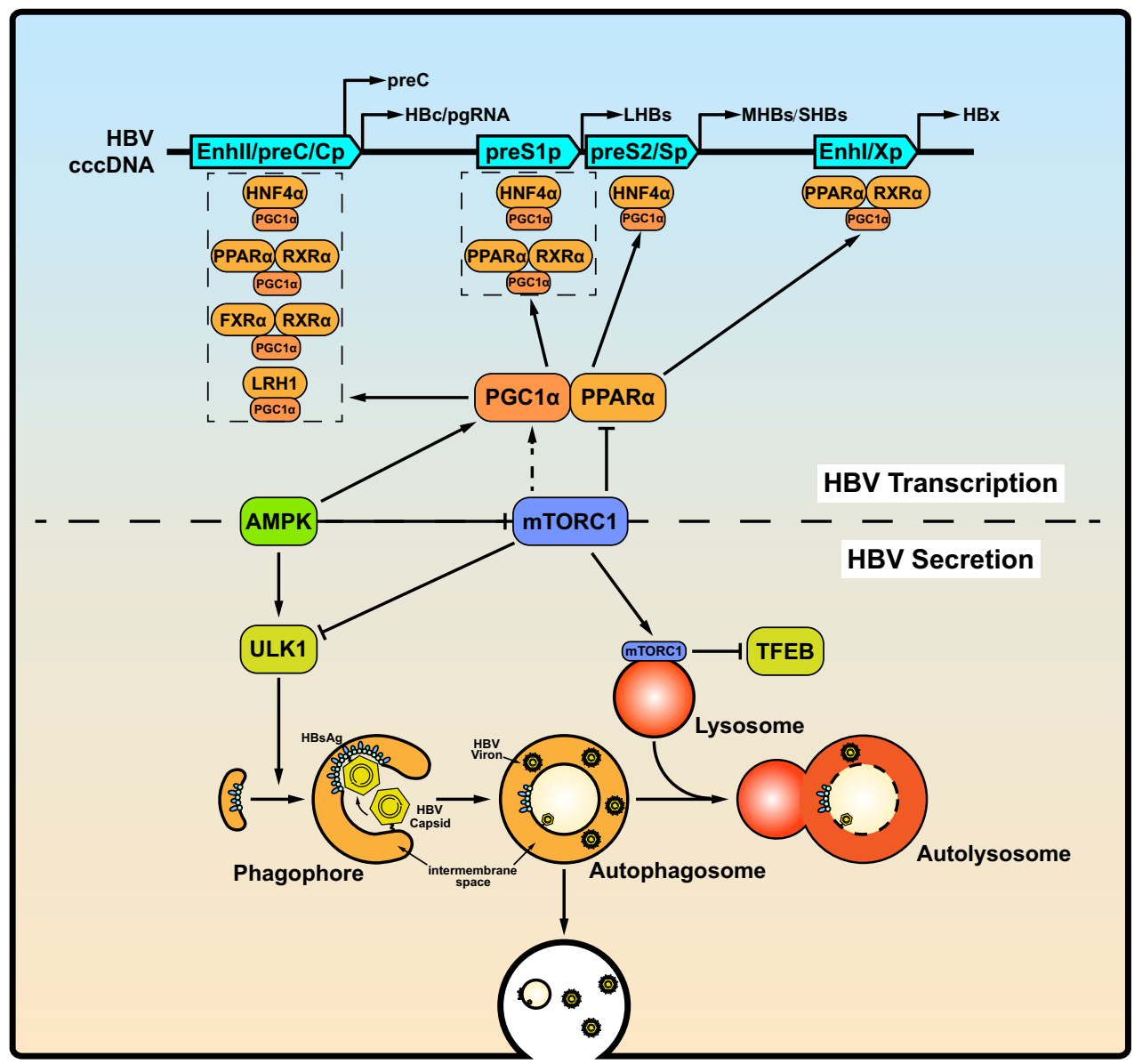

that glucosamine increases HBV replication by blocking mTOR signaling via a feedback mechanism in a HBV mouse model (Lin et al. 2019c).

To maintain effective autophagic flux, autophagosome formation is linked with autophagosomal-lysosomal fusion and lysosomal degradation activity. Although mTOR regulates both early (autophagosome formation) and late (lysosomal activity) phase of autophagy, HBV assembly and release through enhanced autophagy are still beneficial for the production HBV particles despite the degradation by lysosomes. Recent findings also showed that HBV replication can interfere with the lysosomal functions and thereby evade the autophagic degradation process (Xie et al. 2016; Lin et al. 2019a, 2019b). A part of autophagosomes may not fuse with lysosomes for degradation, but rather release HBV proteins and virions through other pathways, such as fusing with MVBs (Wang et al. 2021) or exosomes as indicated by our unpublished work.

As mTOR pathway integrates many different signals, indirect crosstalk with some other major pathways may occur, thereby regulating downstream processes and HBV replication. For example, type I interferon signaling has been shown to crosstalk with Akt/mTOR signaling (Uddin et al. 1995; Lekmine et al. 2004). Our unpublished data revealed that type I interferon may modulate autophagy and subsequently HBV replication via Akt/mTOR pathway.

\section{Conclusions and Perspectives}

In this review, we have summarized recent progress from studies on the interaction between the cellular mTOR signaling pathway and HBV replication. mTOR first attracted the attention of researchers because it may play important roles in the development of HBV-associated HCC. The mTOR inhibitors are able to suppress cell growth in vitro and in vivo. Viral infections often alter host cellular metabolisms through regulating various cellular factors and pathways, including the PI3K/Akt/mTOR signaling pathway. The mTOR signaling pathway is essential in hepatocyte glucose and lipid metabolism, which are two important biological processes for efficient HBV replication. Additionally, mTOR negatively regulates autophagosome formation by inactivating the ULK kinase complex bound to the PIK3C3/BECN1/ATG14 complex and thereby reducing HBV assembly and release through autophagy. Up to date, the mTOR-mediated signaling pathway has been shown to be essential for HBV 
replication and pathogenesis in cell culture models. However, only few studies have been conducted in human tissues or animal models and need to be continued in the future. In addition, there are limitations in studying the cross-regulation between HBV infection and mTOR signaling in human tissues or animal models. The development of new technologies in molecular and cell biology will help to better understand the cross-regulation between HBV infection and mTOR signaling. In the future, this may help identify new therapeutic targeting.

Different concentrations of PI3K, Akt and mTOR inhibitors have different effects on HBV transcription and replication. Guo et al. showed that treatment of HBVexpressing HepG2.2.15 cells with high concentrations of inhibitors of PI3K, Akt, and mTOR (10, 5, and $10 \mu \mathrm{mol} / \mathrm{L}$, respectively) increased the transcription of $3.5-\mathrm{kb}$ and 2.4$\mathrm{kb}$ viral RNA as well as the replication of HBV DNA (Guo et al. 2007b). However, some other studies including ours showed that low concentrations $(\leq 1 \mu \mathrm{mol} / \mathrm{L})$ of the inhibitors significantly enhanced HBV replication through enhancing autophagy instead of HBV transcription (Huang et al. 2014; Lin et al. 2020). There are many binding sites of ubiquitous and hepatocyte-enriched transcription factors in the promoter and enhancer regions of the HBV genome, including that for the transcription factors PGC1 $\alpha$ and the PPAR $\alpha /$ retinoid X receptor alpha $(\mathrm{RXR} \alpha)$ complex. Previously evidences suggested that mTOR could regulate the activity of PGC1 $\alpha$ and PPAR in vitro or in vivo (Raney et al. 1997; Shlomai et al. 2006; Shlomai and Shaul 2009; Reese et al. 2011; Weng et al. 2014), which play essential roles in HBV transcription. Moreover, PGC1 $\alpha$ can be recruited by nuclear receptors (Fig. 2, top), including hepatocyte nuclear factor $4 \alpha(\mathrm{HNF} 4 \alpha)$, the PPAR $\alpha / \mathrm{RXR} \alpha$ heterodimer, the $\mathrm{FXR} \alpha / \mathrm{RXR} \alpha$ heterodimer, and liver receptor homolog 1 (LRH1), which positively regulate HBV pgRNA synthesis to enhance HBV replication (Reese et al. 2011; Weng et al. 2014). Therefore, high-concentrations of PI3K, Akt, and mTOR inhibitors may have a stronger regulatory effect on these transcription factors, while low-concentrations of these inhibitors mainly regulate the mTOR-induced autophagy pathway and have lower regulatory effects on these transcription factors. However, this hypothesis needs to be tested in future experimental approaches. It is likely that these nutrient-regulated transcription factors on HBV transcription also are regulated by HBV activated mTORC1.

Recently, increasing evidence has indicated that the mTOR signaling pathway plays multiple roles in immunity (Weichhart et al. 2015). The interaction of S6K and STING can regulate the activation of IRF3 to induce innate immunity (Wang et al. 2016). The mTOR signaling pathway can regulate c-Fos expression (He et al. 2015), and IFN $\alpha / \beta$ production (Cao et al. 2008). Besides, Delgoffe et al. reported that naïve T cells fail to differentiate into Th1, and Th17 cells in mTOR or Rheb gene knockout mice (Delgoffe et al. 2009, 2011). In addition, rapamycin at a high dose can attenuate $\mathrm{CD} 8^{+} \mathrm{T}$ cell responses (Pearce et al. 2009), and exerts immunostimulatory effects on memory $\mathrm{CD}^{+} \mathrm{T}$ cell differentiation (Araki et al. 2010). Thus, mTOR is involved in the regulation of both innate and adaptive immunity. It is tempting to speculate that mTOR-mediated innate or adaptive immunity may contribute to control HBV infection. Importantly, further understanding of the immunity and mTOR signaling may provide novel targets for regulating immunity and treating HBV-related disease.

Despite recent advances in drug discovery and development, effective antiviral drugs against HBV are still very limited, especially for the cure of chronic HBV infection. Thus, the development of effective, well-tolerated and affordable antiviral treatments is necessary and urgent. The HBV-induced abnormalities in the expression of PI3K/Akt/ mTOR and its downstream regulators and alteration in host metabolism make mTOR a potential target for drug development.

Acknowledgements This work was supported by a scholarship from the Medical Faculty of University Duisburg-Essen, the Foundation for Innovative Research Groups of Hubei Provincial Natural Science Foundation (2018CFA031), Hubei Province's Outstanding Medical Academic Leader Program, the National Natural Science Foundation of China (No.81974079), and the Key R\&D Program of Hunan province (No.2020SK30291).

Funding Open Access funding enabled and organized by Projekt DEAL.

\section{Compliance with Ethical Standards}

Conflict of interest The authors declare that they have no conflict of interest.

Animal and Human Rights Statement This article does not contain any studies with human or animal subjects performed by any of the authors.

Open Access This article is licensed under a Creative Commons Attribution 4.0 International License, which permits use, sharing, adaptation, distribution and reproduction in any medium or format, as long as you give appropriate credit to the original author(s) and the source, provide a link to the Creative Commons licence, and indicate if changes were made. The images or other third party material in this article are included in the article's Creative Commons licence, unless indicated otherwise in a credit line to the material. If material is not included in the article's Creative Commons licence and your intended use is not permitted by statutory regulation or exceeds the permitted use, you will need to obtain permission directly from the copyright holder. To view a copy of this licence, visit http://creativecommons. org/licenses/by/4.0/. 


\section{References}

Araki K, Youngblood B, Ahmed R (2010) The role of mTOR in memory CD8 T-cell differentiation. Immunol Rev 235:234-243

Betz C, Hall MN (2013) Where is mTOR and what is it doing there? J Cell Biol 203:563-574

Bossler F, Hoppe-Seyler K, Hoppe-Seyler F (2019) PI3K/AKT/ mTOR signaling regulates the virus/host cell crosstalk in HPVpositive cervical cancer cells. Int J Mol Sci 20:2188

Cao W, Manicassamy S, Tang H, Kasturi SP, Pirani A, Murthy N, Pulendran B (2008) Toll-like receptor-mediated induction of type I interferon in plasmacytoid dendritic cells requires the rapamycin-sensitive $\mathrm{PI}(3) \mathrm{K}-\mathrm{mTOR}-\mathrm{p} 70 \mathrm{~S} 6 \mathrm{~K}$ pathway. Nat Immunol 9:1157-1164

Cha MY, Kim CM, Park YM, Ryu WS (2004) Hepatitis B virus X protein is essential for the activation of Wnt/beta-catenin signaling in hepatoma cells. Hepatology 39:1683-1693

Chan C, Thurnherr T, Wang J, Gallart-Palau X, Sze SK, Rozen S, Lee CG (2016) Global re-wiring of p53 transcription regulation by the hepatitis B virus X protein. Mol Oncol 10:1183-1195

Chan C, Wang Y, Chow PK, Chung AY, Ooi LL, Lee CG (2013) Altered binding site selection of p53 transcription cassettes by hepatitis B virus X protein. Mol Cell Biol 33:485-497

Chen M, Lu J, Wei W, Lv Y, Zhang X, Yao Y, Wang L, Ling T, Zou $\mathrm{X}$ (2018) Effects of proton pump inhibitors on reversing multidrug resistance via downregulating V-ATPases/PI3K/Akt/ mTOR/HIF-1alpha signaling pathway through TSC1/2 complex and Rheb in human gastric adenocarcinoma cells in vitro and in vivo. Onco Targets Ther 11:6705-6722

Chen X, Hu Y, Zhang W, Chen K, Hu J, Li X, Liang L, Cai X, Hu J, Wang K, Huang A, Tang N (2019) Cisplatin induces autophagy to enhance hepatitis B virus replication via activation of ROS/ JNK and inhibition of the Akt/mTOR pathway. Free Radic Biol Med 131:225-236

Cheng X, Wang Y, Tian J, Zhou L, Chen X, Guo H, Zeng J, Shen N, Li J, Ke J, Zhu Y, Gong J, Chang J, Liu L, Zhong R (2019) SLC10A1 S267F variant influences susceptibility to HBV infection and reduces cholesterol level by impairing bile acid uptake. J Viral Hepat 26:1178-1185

Choi YM, Lee SY, Kim BJ (2019) Naturally occurring hepatitis B virus mutations leading to endoplasmic reticulum stress and their contribution to the progression of hepatocellular carcinoma. Int $\mathbf{J}$ Mol Sci 20: 597

Chung TW, Lee YC, Kim CH (2004) Hepatitis B viral HBx induces matrix metalloproteinase-9 gene expression through activation of ERK and PI-3K/AKT pathways: involvement of invasive potential. FASEB J 18:1123-1125

Chung TW, Lee YC, Ko JH, Kim CH (2003) Hepatitis B Virus X protein modulates the expression of PTEN by inhibiting the function of $\mathrm{p} 53$, a transcriptional activator in liver cells. Cancer Res 63:3453-3458

Ciuffreda L, Di Sanza C, Incani UC, Milella M (2010) The mTOR pathway: a new target in cancer therapy. Curr Cancer Drug Targets 10:484-495

Claus C, Liebert UG (2014) A renewed focus on the interplay between viruses and mitochondrial metabolism. Arch Virol 159:1267-1277

Cunningham JT, Rodgers JT, Arlow DH, Vazquez F, Mootha VK, Puigserver P (2007) mTOR controls mitochondrial oxidative function through a YY1-PGC-1alpha transcriptional complex. Nature 450:736-740

Daud M, Rana MA, Husnain T, Ijaz B (2017) Modulation of Wnt signaling pathway by hepatitis $\mathrm{B}$ virus. Arch Virol 162:2937-2947
Delgoffe GM, Kole TP, Zheng Y, Zarek PE, Matthews KL, Xiao B, Worley PF, Kozma SC, Powell JD (2009) The mTOR kinase differentially regulates effector and regulatory $\mathrm{T}$ cell lineage commitment. Immunity 30:832-844

Delgoffe GM, Pollizzi KN, Waickman AT, Heikamp E, Meyers DJ, Horton MR, Xiao B, Worley PF, Powell JD (2011) The kinase mTOR regulates the differentiation of helper T cells through the selective activation of signaling by mTORC 1 and mTORC 2 . Nat Immunol 12:295-303

Duvel K, Yecies JL, Menon S, Raman P, Lipovsky AI, Souza AL, Triantafellow E, Ma Q, Gorski R, Cleaver S, Vander Heiden MG, MacKeigan JP, Finan PM, Clish CB, Murphy LO, Manning BD (2010) Activation of a metabolic gene regulatory network downstream of mTOR complex 1. Mol Cell 39:171-183

Eaton HE, Saffran HA, Wu FW, Quach K, Smiley JR (2014) Herpes simplex virus protein kinases US3 and UL13 modulate VP11/12 phosphorylation, virion packaging, and phosphatidylinositol 3-kinase/Akt signaling activity. J Virol 88:7379-7388

Efeyan A, Zoncu R, Sabatini DM (2012) Amino acids and mTORC1: from lysosomes to disease. Trends Mol Med 18:524-533

Fang Y, Vilella-Bach M, Bachmann R, Flanigan A, Chen J (2001) Phosphatidic acid-mediated mitogenic activation of mTOR signaling. Science 294:1942-1945

Fanning GC, Zoulim F, Hou J, Bertoletti A (2019) Therapeutic strategies for hepatitis B virus infection: towards a cure. Nat Rev Drug Discov 18:827-844

Feng Z, Zhang H, Levine AJ, Jin S (2005) The coordinate regulation of the p53 and mTOR pathways in cells. Proc Natl Acad Sci U S A 102:8204-8209

Forbes SA, Bindal N, Bamford S, Cole C, Kok CY, Beare D, Jia M, Shepherd R, Leung K, Menzies A, Teague JW, Campbell PJ, Stratton MR, Futreal PA (2011) COSMIC: mining complete cancer genomes in the Catalogue of Somatic Mutations in Cancer. Nucleic Acids Res 39:D945-950

Geier A (2014) Hepatitis B virus: the "metabolovirus" highjacks cholesterol and bile acid metabolism. Hepatology 60:1458-1460

Gish RG, Given BD, Lai CL, Locarnini SA, Lau JY, Lewis DL, Schluep T (2015) Chronic hepatitis B: virology, natural history, current management and a glimpse at future opportunities. Antiviral Res 121:47-58

Glebe D, Bremer CM (2013) The molecular virology of hepatitis B virus. Semin Liver Dis 33:103-112

Groenewoud MJ, Zwartkruis FJ (2013) Rheb and Rags come together at the lysosome to activate mTORC1. Biochem Soc Trans 41:951-955

Guo H, Jiang D, Zhou T, Cuconati A, Block TM, Guo JT (2007a) Characterization of the intracellular deproteinized relaxed circular DNA of hepatitis B virus: an intermediate of covalently closed circular DNA formation. J Virol 81:12472-12484

Guo H, Zhou T, Jiang D, Cuconati A, Xiao GH, Block TM, Guo JT (2007b) Regulation of hepatitis B virus replication by the phosphatidylinositol 3-kinase-akt signal transduction pathway. J Virol 81:10072-10080

Guo Z, Cheng X, Feng X, Zhao K, Zhang M, Yao R, Chen Y, Wang Y, Hao H, Wang Z (2019) The mTORC1/4EBP1/PPARgamma axis mediates insulin-induced lipogenesis by regulating lipogenic gene expression in bovine mammary epithelial cells. J Agric Food Chem 67:6007-6018

Gwinn DM, Shackelford DB, Egan DF, Mihaylova MM, Mery A, Vasquez DS, Turk BE, Shaw RJ (2008) AMPK phosphorylation of raptor mediates a metabolic checkpoint. Mol Cell 30:214-226

Han J, Li E, Chen L, Zhang Y, Wei F, Liu J, Deng H, Wang Y (2015) The CREB coactivator CRTC2 controls hepatic lipid metabolism by regulating SREBP1. Nature 524:243-246

Hay N, Sonenberg N (2004) Upstream and downstream of mTOR. Genes Dev 18:1926-1945 
He L, Zang A, Du M, Ma D, Yuan C, Zhou C, Mu J, Shi H, Li D, Huang X, Deng Q, Xiao J, Yan H, Hui L, Lan K, Xiong S, Li X, Huang Z, Xiao H (2015) mTOR regulates TLR-induced c-fos and Th1 responses to $\mathrm{HBV}$ and $\mathrm{HCV}$ vaccines. Virol Sin 30:174-189

He Q, Song X, Huang Y, Huang W, Ye B, Luo H, Luo H, Wu L, Wang Z, Chen W, Zhang L (2018) Dexamethasone stimulates hepatitis B virus (HBV) replication through autophagy. Med Sci Monit 24:4617-4624

Huang W, Zhao F, Huang Y, Li X, Zhu S, Hu Q, Chen W (2014) Rapamycin enhances HBV production by inducing cellular autophagy. Hepat Mon 14:e20719

Hung JH, Su IJ, Lei HY, Wang HC, Lin WC, Chang WT, Huang W, Chang WC, Chang YS, Chen CC, Lai MD (2004) Endoplasmic reticulum stress stimulates the expression of cyclooxygenase-2 through activation of NF-kappaB and pp38 mitogen-activated protein kinase. J Biol Chem 279:46384-46392

Inoki K, Li Y, Xu T, Guan KL (2003a) Rheb GTPase is a direct target of TSC2 GAP activity and regulates mTOR signaling. Genes Dev 17:1829-1834

Inoki $\mathrm{K}$, Ouyang $\mathrm{H}$, Zhu T, Lindvall $\mathrm{C}$, Wang $\mathrm{Y}$, Zhang $\mathrm{X}$, Yang $\mathrm{Q}$, Bennett C, Harada Y, Stankunas K, Wang CY, He X, MacDougald OA, You M, Williams BO, Guan KL (2006) TSC2 integrates Wnt and energy signals via a coordinated phosphorylation by AMPK and GSK3 to regulate cell growth. Cell 126:955-968

Inoki K, Zhu T, Guan KL (2003b) TSC2 mediates cellular energy response to control cell growth and survival. Cell 115:577-590

Jacinto E, Loewith R, Schmidt A, Lin S, Ruegg MA, Hall A, Hall MN (2004) Mammalian TOR complex 2 controls the actin cytoskeleton and is rapamycin insensitive. Nat Cell Biol 6:1122-1128

Jager S, Handschin C, St-Pierre J, Spiegelman BM (2007) AMPactivated protein kinase (AMPK) action in skeletal muscle via direct phosphorylation of PGC-1alpha. Proc Natl Acad Sci U S A 104:12017-12022

Ji WT, Liu HJ (2008) PI3K-Akt signaling and viral infection. Recent Pat Biotechnol 2:218-226

Karayiannis P (2017) Hepatitis B virus: virology, molecular biology, life cycle and intrahepatic spread. Hepatol Int 11:500-508

Kim DH, Sarbassov DD, Ali SM, King JE, Latek RR, ErdjumentBromage H, Tempst P, Sabatini DM (2002) mTOR interacts with raptor to form a nutrient-sensitive complex that signals to the cell growth machinery. Cell 110:163-175

Kim J, Kundu M, Viollet B, Guan KL (2011) AMPK and mTOR regulate autophagy through direct phosphorylation of Ulk1. Nat Cell Biol 13:132-141

Kim K, Kim KH, Kim HH, Cheong J (2008) Hepatitis B virus X protein induces lipogenic transcription factor SREBP1 and fatty acid synthase through the activation of nuclear receptor LXRalpha. Biochem J 416:219-230

Kim KH, Shin HJ, Kim K, Choi HM, Rhee SH, Moon HB, Kim HH, Yang US, Yu DY, Cheong J (2007) Hepatitis B virus X protein induces hepatic steatosis via transcriptional activation of SREBP1 and PPARgamma. Gastroenterology 132:1955-1967

Kock J, Rosler C, Zhang JJ, Blum HE, Nassal M, Thoma C (2010) Generation of covalently closed circular DNA of hepatitis B viruses via intracellular recycling is regulated in a virus specific manner. PLoS Pathog 6:e1001082

Laplante M, Sabatini DM (2012) mTOR signaling in growth control and disease. Cell 149:274-293

Lee DF, Kuo HP, Chen CT, Hsu JM, Chou CK, Wei Y, Sun HL, Li LY, Ping B, Huang WC, He X, Hung JY, Lai CC, Ding Q, Su JL, Yang JY, Sahin AA, Hortobagyi GN, Tsai FJ, Tsai CH, Hung MC (2007) IKK beta suppression of TSC1 links inflammation and tumor angiogenesis via the mTOR pathway. Cell $130: 440-455$
Lee JO, Kwun HJ, Jung JK, Choi KH, Min DS, Jang KL (2005) Hepatitis B virus $X$ protein represses E-cadherin expression via activation of DNA methyltransferase 1. Oncogene 24:6617-6625

Lee SG, Rho HM (2000) Transcriptional repression of the human p53 gene by hepatitis B viral X protein. Oncogene 19:468-471

Lekmine F, Sassano A, Uddin S, Smith J, Majchrzak B, Brachmann SM, Hay N, Fish EN, Platanias LC (2004) Interferon-gamma engages the p70 S6 kinase to regulate phosphorylation of the 40S S6 ribosomal protein. Exp Cell Res 295:173-182

Li H, Zhu W, Zhang L, Lei H, Wu X, Guo L, Chen X, Wang Y, Tang $\mathrm{H}$ (2015) The metabolic responses to hepatitis B virus infection shed new light on pathogenesis and targets for treatment. Sci Rep $5: 8421$

Li J, Liu Y, Wang Z, Liu K, Wang Y, Liu J, Ding H, Yuan Z (2011) Subversion of cellular autophagy machinery by hepatitis B virus for viral envelopment. J Virol 85:6319-6333

Li Z, Xu G, Qin Y, Zhang C, Tang H, Yin Y, Xiang X, Li Y, Zhao J, Mulholland M, Zhang W (2014) Ghrelin promotes hepatic lipogenesis by activation of mTOR-PPARgamma signaling pathway. Proc Natl Acad Sci U S A 111:13163-13168

Liang TJ, Block TM, McMahon BJ, Ghany MG, Urban S, Guo JT, Locarnini S, Zoulim F, Chang KM, Lok AS (2015) Present and future therapies of hepatitis B: from discovery to cure. Hepatology 62:1893-1908

Lin Y, Deng W, Pang J, Kemper T, Hu J, Yin J, Zhang J, Lu M (2017) The microRNA-99 family modulates hepatitis B virus replication by promoting IGF-1R/PI3K/Akt/mTOR/ULK1 signaling-induced autophagy. Cell Microbiol. https://doi.org/10.1111/cmi. 12709

Lin Y, Wu C, Wang X, Kemper T, Squire A, Gunzer M, Zhang J, Chen X, Lu M (2019a) Hepatitis B virus is degraded by autophagosome-lysosome fusion mediated by Rab7 and related components. Protein Cell 10:60-66

Lin Y, Wu C, Wang X, Liu S, Kemper T, Li F, Squire A, Zhu Y, Zhang J, Chen X, Lu M (2019b) Synaptosomal-associated protein 29 is required for the autophagic degradation of hepatitis B virus. FASEB J 33:6023-6034

Lin Y, Wu C, Wang X, Liu S, Zhao K, Kemper T, Yu H, Li M, Zhang J, Chen M, Zhu Y, Chen X, Lu M (2019c) Glucosamine promotes hepatitis $\mathrm{B}$ virus replication through its dual effects in suppressing autophagic degradation and inhibiting MTORC1 signaling. Autophagy 16:568-516

Lin Y, Zhao Z, Huang A, Lu M (2020) Interplay between cellular autophagy and hepatitis B virus replication: a systematic review. Cells 9: 2101

Liu B, Fang M, He Z, Cui D, Jia S, Lin X, Xu X, Zhou T, Liu W (2015) Hepatitis B virus stimulates G6PD expression through HBx-mediated Nrf2 activation. Cell Death Dis 6:e1980

Liu H, Xu J, Zhou L, Yun X, Chen L, Wang S, Sun L, Wen Y, Gu J (2011) Hepatitis B virus large surface antigen promotes liver carcinogenesis by activating the Src/PI3K/Akt pathway. Cancer Res 71:7547-7557

Liu Y, Zhang DT, Liu XG (2015) mTOR signaling in T cell immunity and autoimmunity. Int Rev Immunol 34:50-66

Ma L, Chen Z, Erdjument-Bromage H, Tempst P, Pandolfi PP (2005) Phosphorylation and functional inactivation of TSC2 by Erk implications for tuberous sclerosis and cancer pathogenesis. Cell 121:179-193

Mehta A, Lu X, Block TM, Blumberg BS, Dwek RA (1997) Hepatitis $B$ virus (HBV) envelope glycoproteins vary drastically in their sensitivity to glycan processing: evidence that alteration of a single N-linked glycosylation site can regulate HBV secretion. Proc Natl Acad Sci U S A 94:1822-1827

Na TY, Shin YK, Roh KJ, Kang SA, Hong I, Oh SJ, Seong JK, Park CK, Choi YL, Lee MO (2009) Liver X receptor mediates hepatitis B virus $\mathrm{X}$ protein-induced lipogenesis in hepatitis B 
virus-associated hepatocellular carcinoma. Hepatology 49:1122-1131

Nassal M (2015) HBV cccDNA: viral persistence reservoir and key obstacle for a cure of chronic hepatitis B. Gut 64:1972-1984

Oehler N, Volz T, Bhadra OD, Kah J, Allweiss L, Giersch K, Bierwolf J, Riecken K, Pollok JM, Lohse AW, Fehse B, Petersen J, Urban S, Lutgehetmann M, Heeren J, Dandri M (2014) Binding of hepatitis B virus to its cellular receptor alters the expression profile of genes of bile acid metabolism. Hepatology 60:1483-1493

Patman G (2014) Hepatitis: HBV infection alters bile acid metabolism gene profile. Nat Rev Gastroenterol Hepatol 11:332

Pearce EL, Walsh MC, Cejas PJ, Harms GM, Shen H, Wang LS, Jones RG, Choi Y (2009) Enhancing CD8 T-cell memory by modulating fatty acid metabolism. Nature 460:103-107

Peterson TR, Sengupta SS, Harris TE, Carmack AE, Kang SA, Balderas E, Guertin DA, Madden KL, Carpenter AE, Finck BN, Sabatini DM (2011) mTOR complex 1 regulates lipin 1 localization to control the SREBP pathway. Cell 146:408-420

Ranadheera C, Coombs KM, Kobasa D (2018) Comprehending a killer: the Akt/mTOR signaling pathways are temporally highjacked by the highly pathogenic 1918 Influenza Virus. EBioMedicine 32:142-163

Raney AK, Johnson JL, Palmer CN, McLachlan A (1997) Members of the nuclear receptor superfamily regulate transcription from the hepatitis B virus nucleocapsid promoter. J Virol $71: 1058-1071$

Rawat S, Bouchard MJ (2015) The hepatitis B virus (HBV) HBx protein activates AKT to simultaneously regulate HBV replication and hepatocyte survival. J Virol 89:999-1012

Reese V, Ondracek C, Rushing C, Li L, Oropeza CE, McLachlan A (2011) Multiple nuclear receptors may regulate hepatitis B virus biosynthesis during development. Int $\mathrm{J}$ Biochem Cell Biol 43:230-237

Roux PP, Ballif BA, Anjum R, Gygi SP, Blenis J (2004) Tumorpromoting phorbol esters and activated Ras inactivate the tuberous sclerosis tumor suppressor complex via $\mathrm{p} 90$ ribosomal S6 kinase. Proc Natl Acad Sci U S A 101:13489-13494

Sarbassov DD, Ali SM, Kim DH, Guertin DA, Latek RR, ErdjumentBromage H, Tempst P, Sabatini DM (2004) Rictor, a novel binding partner of mTOR, defines a rapamycin-insensitive and raptor-independent pathway that regulates the cytoskeleton. Curr Biol 14:1296-1302

Sarbassov DD, Guertin DA, Ali SM, Sabatini DM (2005) Phosphorylation and regulation of $\mathrm{Akt} / \mathrm{PKB}$ by the rictor-mTOR complex. Science 307:1098-1101

Saxton RA, Sabatini DM (2017) mTOR signaling in growth, metabolism, and disease. Cell 168:960-976

Semenza GL (2010) HIF-1: upstream and downstream of cancer metabolism. Curr Opin Genet Dev 20:51-56

Sengupta S, Peterson TR, Laplante M, Oh S, Sabatini DM (2010) mTORC1 controls fasting-induced ketogenesis and its modulation by ageing. Nature 468:1100-1104

Shimobayashi M, Hall MN (2014) Making new contacts: the mTOR network in metabolism and signalling crosstalk. Nat Rev Mol Cell Biol 15:155-162

Shin HJ, Park YH, Kim SU, Moon HB, Park DS, Han YH, Lee CH, Lee DS, Song IS, Lee DH, Kim M, Kim NS, Kim DG, Kim JM, Kim SK, Kim YN, Kim SS, Choi CS, Kim YB, Yu DY (2011) Hepatitis B virus $X$ protein regulates hepatic glucose homeostasis via activation of inducible nitric oxide synthase. J Biol Chem 286:29872-29881

Shlomai A, Paran N, Shaul Y (2006) PGC-1alpha controls hepatitis B virus through nutritional signals. Proc Natl Acad Sci U S A 103:16003-16008
Shlomai A, Shaul Y (2009) The metabolic activator FOXO1 binds hepatitis B virus DNA and activates its transcription. Biochem Biophys Res Commun 381:544-548

Sigrid Schmitt DG, Alving K, Tolle TK, Linder M, Geyer H, Linder D, Peter-Katalinic J, Gerlich WH, Geyeri R (1999) Analysis of the Pre-S2 N- and O-linked glycans of the M surface protein from human hepatitis B virus. J Biol Chem 274:11945-11957

Sir D, Tian Y, Chen WL, Ann DK, Yen TS, Ou JH (2010) The early autophagic pathway is activated by hepatitis B virus and required for viral DNA replication. Proc Natl Acad Sci U S A 107:4383-4388

Subic M, Zoulim F (2018) How to improve access to therapy in hepatitis B patients. Liver Int 38(Suppl 1):115-121

Tang LSY, Covert E, Wilson E, Kottilil S (2018) Chronic hepatitis B infection: A review. JAMA 319:1802-1813

Tarn C, Lee S, Hu Y, Ashendel C, Andrisani OM (2001) Hepatitis B virus $\mathrm{X}$ protein differentially activates RAS-RAF-MAPK and JNK pathways in X-transforming versus non-transforming AML12 hepatocytes. J Biol Chem 276:34671-34680

Tee AR, Manning BD, Roux PP, Cantley LC, Blenis J (2003) Tuberous sclerosis complex gene products, Tuberin and Hamartin, control mTOR signaling by acting as a GTPaseactivating protein complex toward Rheb. Curr Biol 13:1259-1268

Teng CF, Hsieh WC, Wu HC, Lin YJ, Tsai HW, Huang W, Su IJ (2015a) Hepatitis B virus pre-S2 mutant induces aerobic glycolysis through mammalian target of rapamycin signal cascade. PLoS ONE 10:e122373

Teng CF, Wu HC, Hsieh WC, Tsai HW, Su IJ (2015b) Activation of ATP citrate lyase by mTOR signal induces disturbed lipid metabolism in hepatitis B virus pre-S2 mutant tumorigenesis. J Virol 89:605-614

Teng CF, Wu HC, Tsai HW, Shiah HS, Huang W, Su IJ (2011) Novel feedback inhibition of surface antigen synthesis by mammalian target of rapamycin (mTOR) signal and its implication for hepatitis B virus tumorigenesis and therapy. Hepatology 54:1199-1207

Tian Y, Sir D, Kuo CF, Ann DK, Ou JH (2011) Autophagy required for hepatitis B virus replication in transgenic mice. J Virol 85:13453-13456

Tong S, Revill P (2016) Overview of hepatitis B viral replication and genetic variability. J Hepatol 64:S4-16

Uddin S, Yenush L, Sun XJ, Sweet ME, White MF, Platanias LC (1995) Interferon-alpha engages the insulin receptor substrate-1 to associate with the phosphatidylinositol 3'-kinase. J Biol Chem 270:15938-15941

Wang BT, Ducker GS, Barczak AJ, Barbeau R, Erle DJ, Shokat KM (2011) The mammalian target of rapamycin regulates cholesterol biosynthetic gene expression and exhibits a rapamycin-resistant transcriptional profile. Proc Natl Acad Sci U S A 108:15201-15206

Wang F, Alain T, Szretter KJ, Stephenson K, Pol JG, Atherton MJ, Hoang HD, Fonseca BD, Zakaria C, Chen L, Rangwala Z, Hesch A, Chan ESY, Tuinman C, Suthar MS, Jiang Z, Ashkar AA, Thomas G, Kozma SC, Gale M Jr, Fitzgerald KA, Diamond MS, Mossman K, Sonenberg N, Wan Y, Lichty BD (2016) S6KSTING interaction regulates cytosolic DNA-mediated activation of the transcription factor IRF3. Nat Immunol 17:514-522

Wang HC, Wu HC, Chen CF, Fausto N, Lei HY, Su IJ (2003) Different types of ground glass hepatocytes in chronic hepatitis $B$ virus infection contain specific pre-S mutants that may induce endoplasmic reticulum stress. Am J Pathol 163:2441-2449

Wang HY, Yang SL, Liang HF, Li CH (2014) HBx protein promotes oval cell proliferation by up-regulation of cyclin D1 via activation of the MEK/ERK and PI3K/Akt pathways. Int J Mol Sci $15: 3507-3518$ 
Wang J, Chen J, Liu Y, Zeng X, Wei M, Wu S, Xiong Q, Song F, Yuan X, Xiao Y, Cao Y, Li C, Chen L, Guo M, Shi YB, Sun G, Guo D (2019) Hepatitis B Virus Induces Autophagy to Promote its Replication by the Axis of miR-192-3p-XIAP Through NF kappa B Signaling. Hepatology 69:974-992

Wang M, Qiu N, Lu S, Xiu D, Yu J, Wang XT, Lu F, Li T, Liu X, Zhuang H (2013) Serum hepatitis B surface antigen is correlated with intrahepatic total HBV DNA and cccDNA in treatmentnaive patients with chronic hepatitis $B$ but not in patients with HBV related hepatocellular carcinoma. J Med Virol 85:219-227

Wang P, Guo QS, Wang ZW, Qian HX (2013) HBx induces HepG-2 cells autophagy through PI3K/Akt-mTOR pathway. Mol Cell Biochem 372:161-168

Wang X, Huo B, Liu J, Huang X, Zhang S, Feng T (2019) Hepatitis B virus $\mathrm{X}$ reduces hepatocyte apoptosis and promotes cell cycle progression through the $\mathrm{Akt} / \mathrm{mTOR}$ pathway in vivo. Gene 691:87-95

Wang X, Lin Y, Kemper T, Chen J, Yuan Z, Liu S, Zhu Y, Broering R, Lu M (2020a) AMPK and Akt/mTOR signalling pathways participate in glucose-mediated regulation of hepatitis B virus replication and cellular autophagy. Cell Microbiol 22:e13131

Wang X, Lin Y, Liu S, Zhu Y, Lu K, Broering R, Lu M (2020b) O-GlcNAcylation modulates HBV replication through regulating cellular autophagy at multiple levels. FASEB J 34:14473-14489

Wang X, Wei Z, Lan T, He Y, Cheng B, Li R, Chen H, Li F, Liu G, Jiang B, Lin Y, Lu M, Meng Z (2021) CCDC88A/GIV promotes HBV replication and progeny secretion via enhancing endosomal trafficking and blocking autophagic degradation. Autophagy. https://doi.org/10.1080/15548627.2021.1934271:1-18

Weichhart T, Hengstschlager M, Linke M (2015) Regulation of innate immune cell function by mTOR. Nat Rev Immunol 15:599-614

Weng L, Enomoto A, Miyoshi H, Takahashi K, Asai N, Morone N, Jiang P, An J, Kato T, Kuroda K, Watanabe T, Asai M, IshidaTakagishi M, Murakumo Y, Nakashima H, Kaibuchi K, Takahashi M (2014) Regulation of cargo-selective endocytosis by dynamin 2 GTPase-activating protein girdin. EMBO J 33:2098-2112

Wong VW, Wong GL, Chu WC, Chim AM, Ong A, Yeung DK, Yiu KK, Chu SH, Chan HY, Woo J, Chan FK, Chan HL (2012) Hepatitis B virus infection and fatty liver in the general population. J Hepatol 56:533-540

Wullschleger S, Loewith R, Hall MN (2006) TOR signaling in growth and metabolism. Cell 124:471-484

Xiang K, Wang B (2018) Role of the PI3K/AKT/mTOR pathway in hepatitis B virus infection and replication. Mol Med Rep 17:4713-4719
Xie N, Yuan K, Zhou L, Wang K, Chen HN, Lei Y, Lan J, Pu Q, Gao W, Zhang L, Shen G, Li Q, Xiao H, Tang H, Xiang R, He M, Feng P, Nice EC, Wei Y, Zhang H, Yang J, Huang C (2016) PRKAA/AMPK restricts HBV replication through promotion of autophagic degradation. Autophagy 12:1507-1520

Xu X, Fan Z, Kang L, Han J, Jiang C, Zheng X, Zhu Z, Jiao H, Lin J, Jiang K, Ding L, Zhang H, Cheng L, Fu H, Song Y, Jiang Y, Liu J, Wang R, Du N, Ye Q (2013) Hepatitis B virus X protein represses miRNA-148a to enhance tumorigenesis. J Clin Invest 123:630-645

Yan H, Peng B, Liu Y, Xu G, He W, Ren B, Jing Z, Sui J, Li W (2014) Viral entry of hepatitis B and D viruses and bile salts transportation share common molecular determinants on sodium taurocholate cotransporting polypeptide. J Virol 88:3273-3284

Yang F, Yan S, He Y, Wang F, Song S, Guo Y, Zhou Q, Wang Y, Lin Z, Yang Y, Zhang W, Sun S (2008) Expression of hepatitis B virus proteins in transgenic mice alters lipid metabolism and induces oxidative stress in the liver. J Hepatol 48:12-19

Yang JC, Teng CF, Wu HC, Tsai HW, Chuang HC, Tsai TF, Hsu YH, Huang W, Wu LW, Su IJ (2009) Enhanced expression of vascular endothelial growth factor-A in ground glass hepatocytes and its implication in hepatitis B virus hepatocarcinogenesis. Hepatology 49:1962-1971

Yen CJ, Lin YJ, Yen CS, Tsai HW, Tsai TF, Chang KY, Huang WC, Lin PW, Chiang CW, Chang TT (2012) Hepatitis B virus X protein upregulates mTOR signaling through IKKbeta to increase cell proliferation and VEGF production in hepatocellular carcinoma. PLoS ONE 7:e41931

Zhang X, Zhang E, Ma Z, Pei R, Jiang M, Schlaak JF, Roggendorf M, $\mathrm{Lu}$ M (2011) Modulation of hepatitis B virus replication and hepatocyte differentiation by MicroRNA-1. Hepatology 53:1476-1485

Zhou J, Tan SH, Nicolas V, Bauvy C, Yang ND, Zhang J, Xue Y, Codogno P, Shen HM (2013) Activation of lysosomal function in the course of autophagy via mTORC1 suppression and autophagosome-lysosome fusion. Cell Res 23:508-523

Zhu M, Guo J, Li W, Xia H, Lu Y, Dong X, Chen Y, Xie X, Fu S, Li M (2015) HBx induced AFP receptor expressed to activate PI3K/ AKT signal to promote expression of $\mathrm{Src}$ in liver cells and hepatoma cells. BMC Cancer 15:362

Zhu M, Li W, Lu Y, Dong X, Lin B, Chen Y, Zhang X, Guo J, Li M (2017) HBx drives alpha fetoprotein expression to promote initiation of liver cancer stem cells through activating PI3K/AKT signal pathway. Int J Cancer 140:1346-1355 